\title{
VISUAL ARTS AND 2D - 3D MOTION TYPO.GRAPHIC DESIGN
}

Anastasios Maragiannis

School of Architecture and Construction

University of Greenwich

Mansion Site, Bexley Rd, Eltham

London SE9 2PQ

UK

A.Maragiannis@gre.ac.uk

http://www.i4type.com

Abstract - This project explores issues of typographic design within multimedia contexts, which include interactive, sonic and animated components.

\section{INTRODUCTION}

In recent years there have been few actions interested in the engagement of the typographic motion and the visual typographic communication in art and design. Practitioners more recently have been trying to relate motion and sound to typographic elements. Quite few of the screen design practitioners, the theoreticians but also the fine and visual artists, have indicated the fact that this correlation has always provided them with unique consequences. Johana Drucker mentions the fact that the convention of visual typographic experimentation started before the digital era, back in the beginning of printing. According to Richard Lanham in The electronic word, the interaction of the typography and image goes back to the Greek poet Simias from the $4^{\text {th }}$ century BC, when the interaction of type and objects was very common for the expansion of an artistic piece or environment. This paper will try to identify key elements in designing with 2D and 3D Typo.graphic contents for electronic media and visual arts in order to approach and examine environments that could facilitate the design process and expand the communication experience.

\section{Type in Digital Era}

Type started to 'react' and 'interact', as a free physical form, and that happened with the advent of the desktop publishing.

Digital technology radically influenced typographic design beginning in the early 1980s. Charles Bigelow and Donald Day in 1983 identified digital type as that "made up of discrete elements. These elements can be line strokes, pixels, colors, shades of gray, or any other graphic unit from which a letterform can be constructed. Hence, digital typography is not new: mosaic tiles, embroidered samplers, and arrays of lights on theater marquees have long represented alphabetic characters as relatively coarse discrete arrays". However, in focusing on the display device of the cathode-ray tube (CRT), and the requisite "digital computer...needed to control the on-off pattern of the electron beam" that articulated letterforms on the screen, they defined it specifically in 
EVA 2009 London Conference 6-8 July

Anastasios Maragiannis

terms of computer technology [1]. The computer enabled designers to create and manipulate letters in modern ways, offering new options for crafting letterforms and "outputting" them, whether in the medium of toner particles on paper and then as pixels on a screen.

Most digital tools at first required (due to technical constraints) and later explicitly encouraged (due to technical advances) specific kinds of representations that would challenge their historical experiences. Later, in the late 1990s, and now the alteration of letters continues. The chronological opportunities of screen resulted in even more fundamental representations of letterforms that offer expanded formal and stylistic possibilities, while further techniques challenge the medium of reading and writing.

Back in the late 1970s and early 1980s, particularly MIT and Stanford designers' researchers and programmers, began developing pioneering ways to describe and image letters in a digital form [2]. Notably Philippe Coueignoux's CSD (Character Simulated Design) of 1975 decomposed the Roman alphabet into a set of primitives that could be recombined to form any letter. Furthermore, both Pijush Ghosh and Charles Bigelow attempted a similar strategy in 1983. Donald Knuth's ground breaking METAFONT provided a rich programming language for designing type through the algorithmic specification of geometrical relationships [3]. However, the mathematical expression it required was unclear and not recognisable to most typographic designers, and METAFONT never caught on [4]. Digital typography accepted an ever-widening group of districts, from computer scientists such as Knuth to more traditional type designers including Kris Holmes and Charles Bigelow, who were to produce innovations for the page and later for the screen based format. In 1986 they introduce their typeface, Lucida, which satisfied the multiple demands of page and screen through a comprehensive set of fonts suitable for printing and screen display [5].

In 1980 Macintosh introduced the NeXT generation computer, operating PostScript for both screen display and printed output, eliminating any need for intermediate transformation. The NeXT interface, through an expanded range of values from black to white, displayed icons and dialog boxes modelled with greater dimensionality, pushing the visual space of the graphical user interface into different and more experimental pathways expanding the design possibilities for the designers, starting point from 2D to 3D experimental illusions that for the first time will engage the user/designer experience.

The fact that type choice was frequently available on screen, created a contemporary perception of this subject. The Macintosh computer arrived with fonts only for screen base design and not for printing, causing issues such as 'clearness' of type, and typography in new media.

\section{Corresponding Innovations in Graphic Design}

As a result of the new Macintosh arrival, only a small number of perceptive graphic designers recognized the visual possibilities that computer-based typography will offer in the near future. The new technologies in the developed revolution started forming a key change in the understanding of the principles of type, so to will practitioners 
EVA 2009 London Conference 6-8 July

Anastasios Maragiannis

understanding of the principles of typography change as a result of the new electronic revolution.

In 1985, early pioneers such as Zuzana Licko designed three typefaces - Emperor, Oakland, and Emigre - that deliberately exploited the look of the pixel.

Zuzana embraced this new technology, and with the freedom of typographic use started to explore its boundaries in a different and more artistic level. Typefaces soon redefined the look of an emerging publication, Emigre, founded by Licko's husband, Rudy VanderLans, with artist Marc Susan and screenwriter Menno Meyjes. It has since become one of the most influential design publications of this century; serving as a primary vehicle for the dissemination of new critical typographic ideas. Emigre showcased typefaces designed by Licko and others, and served as a catalogue for purchasing those very fonts.

Surprisingly typography could now be stretched, rotated and deformed in every imaginable style.

Following this unique experience and at the same time, in Los Angeles, April Greiman, a Swiss-trained graphic designer, began experimenting extensively with digital imaging and typography in her printed work. Like Licko and VanderLans, she used pixellated letterforms and pictures in posters and brochures, later incorporating video imagery as well [7]. By bringing the actual look of the screen - whether a computer display or television monitor - to paper, Greiman began to challenge the power of the 'white' page as the official owner of the printed word. Silicon Valley's influence transformed her work even further by providing a contemporary more formal vocabulary explicitly formed by visual electronic technology.

While pixellation characterised the glance of these early electronic typographic experiments, antialiasing and blurring characterised the later look of digital typography. For more on the technical aspects and aesthetic and cultural implications of blurring in contemporary graphic design, see Loretta Staples [8]. 'Aliasing' is a technical term used to describe the stair-step appearance of curved edges of forms composed of pixels. In letterforms, aliasing is especially problematic because this stair-stepping interferes with the smoothness of curvature required to define so many individual characters.

At the beginning of the 1990's the creative group Tomato and David Carson started to move forwards the typographic boundaries by researching and revising again the values and principles of typography.

There are similar approaches for the understanding of those principles and can be found in research work of other designers. Rob Carter investigated the morphology and semiotics of print typography. The main goal of his book is to show the utility of semiotics and typography and their progress through computer experimentation. He states that desktop publishing leads to a typographic journey beyond rules. Focusing on semiotics, a similar approach was followed by David Crow, into how visual communication works, which argues that the letters can be presented not only as typographic elements but also as symbols to communicate messages.

Moving to the specifics of typography in new media, Carter supports the idea that design messages influence people. He underscores that morphology (collection of 
EVA 2009 London Conference 6-8 July

Anastasios Maragiannis

typographic factors) guides the reader through explanation to understand the meanings and messages.

In Andy Ellison's book The complete guide to digital type : creative use of typography in the digital arts the author analyses the several ways that practitioners use screen based typeforms for their creations. In this publication it is highlighted that "[t] he internet has allowed designers to create interactive content in ways that were not previously possible" [9]. This ability has been made widespread through the democratization of technology, ease of access to digital tools, which allows designers to produce complicated creations and projects with type that are very difficult with traditional typographic media. An expanded approach of this point will be offered through different researches, including animation, sound and interactivity.

Important work has also been done by Jessica Helfand [10], Ellen Lupton [11], and Max Bruinsna [12], all of whom cover issues such as print and screen-based typeforms as a new language and what is going beyond this.

\section{Typography and Computation Art Technology}

While most graphic designers jumped to take advantage of new opportunities posed by the Web in the mid-1990s, university and industry researchers pursued more innovative and radical approaches to type design in cyberspace as a result of their vast computing resources and funding to permit such exploration. MIT's Visible Language Workshop, under the direction of Muriel Cooper, produced prototypes of multidimensional information displays incorporating type.

Around 1994 a Media Lab research group was headed by Cooper's heir apparent, John Macda. His Aesthetics \& Computation Group explores the connection between typography and programming to exploit computer-processing power. Macda explored print and interactive design, and published whimsical electronic typographic works $[13,14]$ and represents a new breed of designer - the typographer/programmer intended to drive the future of graphic design innovation.

On the other hand, J. Abbott Miller of the design studio Design/Writing/Research undertook innovative experiments in another dimensional typography. Using high-end computer workstations, Miller and his colleagues shaped three-dimensional letterforms [15]. Lathing, extrusion, and texture mapping defined new typefaces as well as novel interpretations of existing classics.

The Internet has given the opportunity for typographic form to be developed through the screen. Not only researchers but also everybody with access to the World Wide Web around the globe use the Internet for sending and receiving. As a result of this transaction, its users generate Web content, and more specifically, typographers use it to collaborate on typeforms and to distribute type. This can be seen in sites such as http://typophile.com and http://www.typotheque.com

"The second generation of web apps is here, and has helped shape the web into an online operating system" [16]. This statement reflects the transformation of the web into a more developed form.

From the other hand practitioners, like Nick Hayes and Jason Arber claim that during the last decade there are opportunities to expand creativity especially using typeforms 
on the Internet. Despite the significant progress in this field there is need for advancing the Internet and motion typographic quality because there is still a substantial lack of scientific research in academic publication and researches.

The letters that make up most alphabets in most languages are designed to be read flat, frontal, and upright. But letters can be animated, and in the process of becoming dynamic typography can take on the intonations and the voice of the spoken word, the emotional characteristics of dance or music, or the narrative qualities of film. The World Wide Web and in particular the network environment offer possibilities and expansions of this typographic role [17].

In the beginning of the new century the web is leading the design process. Primarily practitioners, typographers, and web designers employ the principles of established, traditional print-based typography and simply apply them to the screen. For example, type-fonts designed specifically for the web are being more vigorously developed, named "pixel-fonts"; typeface designed to read and render well at small sizes with no antialiasing, and 2D typography turns into 3D, and spatial arrangements are created.

But with today's contemporary audience and designers, expectations of visual capabilities the time, sound, animation and interaction responses that movement and visual effects can conjure up, are often desired and even expected. Technology makes it easy to create an "optical illusion" and complexity in forms - the addition of animation makes it even easier [18].

According to Philippe Codognet, semiotics can be a useful and powerful tool in order to rephrase information theory and computer technology and shed a new light on this phenomenon. The current success of the World Wide Web protocol on the Internet is due to its ability to manipulate images and sound in addition to simple alphanumeric text.

In the first six years of $21^{\text {st }}$ century, the growth of experimental typography has been enhanced through the advancement of new technologies and the expansion of World Wide Web in connection with new designing software.

The integration of type in motion into a design clarification was now not only accessible to average designers through the medium of the web, but also through the more traditional medium of video.

\section{"Typo.Grafie" in the Visual Arts}

The World Wide Web allows artists/designers to experiment more with type on screen, either as part of visual art or as part of the communication process. The Typeface project (http://i4type.com/typeface) can be seen as tentative because it is trying to showcase different potentials of the letterforms. The term 'tentative' refers to the ways that the typeforms can be used in order to discuss and analyse a series of different approaches. As a process it must also be specified that this first approach of the practice-based research will use these 'experimentations' to outline an additional use of type when employing those forms.

This practice-based research project investigates how technology changed typography and how typography affects the user. It explores the visual and expressive nature of typography in electronic media and takes the spectators on a journey of 
typographic exploration, showing the various uses of typeforms through interactivity, animation and sound.

The Typeface practice-based research project includes eight different experiments with a common objective. All of them try to showcase the different potential of letters and words in the most engaging way, taking the spectator into a journey of typographic exploration, as a communicative function.

1. Experiencing: You are presented with random adjectives and challenged to combine them in a sentence describing you. By printing your work you have the opportunity to observe, evaluate and compare the final outcome. (Figure 2)

2. Feeling: A word of the same meaning can be presented in many ways each time, to evoke a different feeling. The word is always the same but the effect used creates a certain mood. (Figure 3)

3. Inspiring: A word is usually the outcome of a more than two letters combination. A letter can be more than a word it self, it can be an inspiring tool to create a word or it can be an image in its own right. That could be analysed as a prompt for creative engagement. (Figure 4)

4. Differentiating: A word is presented in five different ways by using five different fonts and five different sizes. The same word can create a different feeling, be more playful or formal, more or less significant, by altering fonts and sizes. (Figure 5)

5. Transforming: In real life we base many of our decisions on our aesthetics. In typography we have the potential via technology in order to alter a word's rotation, transparency or size, to extend the traditional boundaries of its appearance. Plasticity of typeform via interaction. (Figure 6)

6. Learning: This visual dictionary unfolds the meaning of the word not only relating to the word's attributes but also to the word's appearance. You learn the meaning of the word by viewing the different styles used to describe it. (Figure 7)

7. 3D Experience: We are used to viewing letters in a $2 \mathrm{~d}$ space, forgetting all the other dimensions. A letter can be presented in a $3 \mathrm{D}$ environment, letting you decide which of its hidden aspects you want to explore. Changing of the viewpoint allows different perceptions and meanings to accrue. (Figure 8)

8. Play: A game could be a way of viewing the differences between static, animated and interactive text. You are able to see all the different ways in which a word can be presented by controlling the final appearance and direction of this word. (Figure 9)

These approaches reflect the nature of the "practice-based" aspects of a research. It is not an existing methodology but an effective synthesis of different approaches, which characterise various perceptions of new media and typography and spectators. This first work, which was produced at an early stage of a $\mathrm{PhD}$ research, was named Typeface in an attempt to specify the different "faces" of the research itself.

Typeface aims to simplify the understanding of the artist, when is creating for the audience and user. 


\section{EVA 2009 London Conference 6-8 July \\ Anastasios Maragiannis}

We are now very much in an era of type in motion. The technologies of the web and the ease of digital video production allow designers to apply effects and treatments to type that were never before available when type was solely print-based.

Designers are now allowed, through the use of technology, to establish new principles for typographic design in these new electronic media; exploring not only the two dimensions of the letter but also other dimensions that will unfold unique design experiences.

\section{ILLUSTRATIONS, FIGURES AND TABLES}

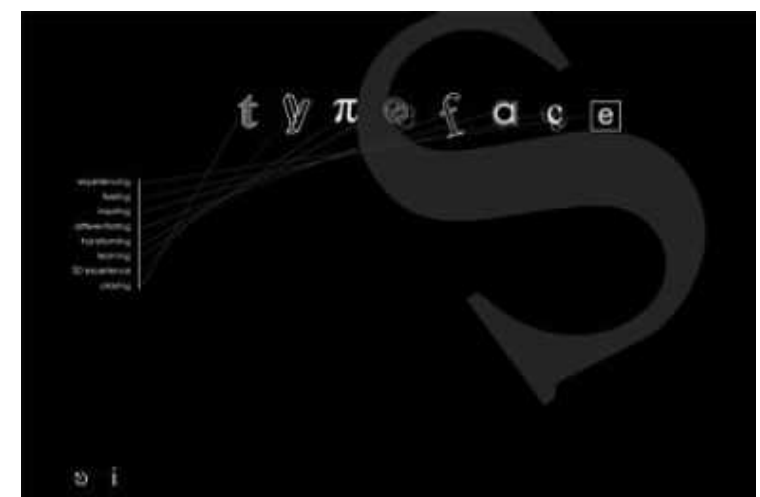

Figure 1. Typeface

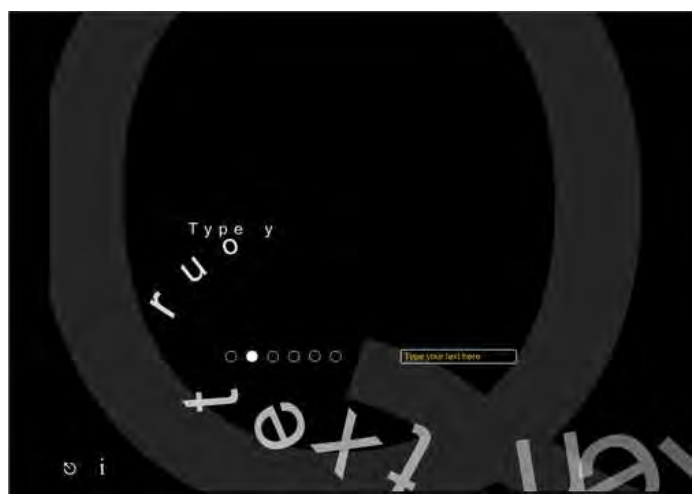

Figure 3. Feeling

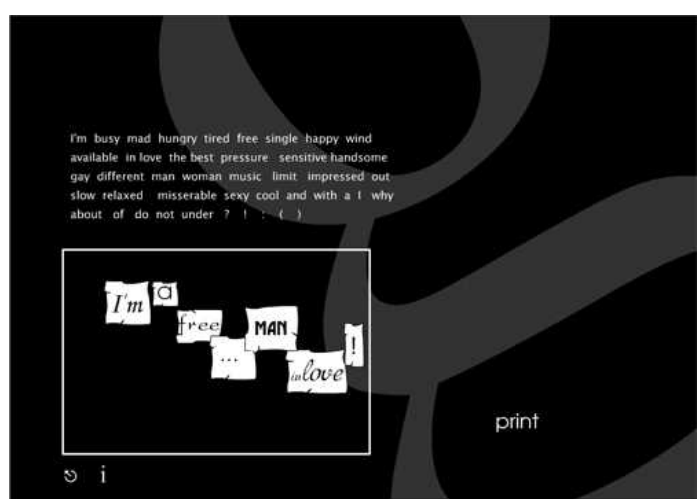

Figure 2. Experiencing

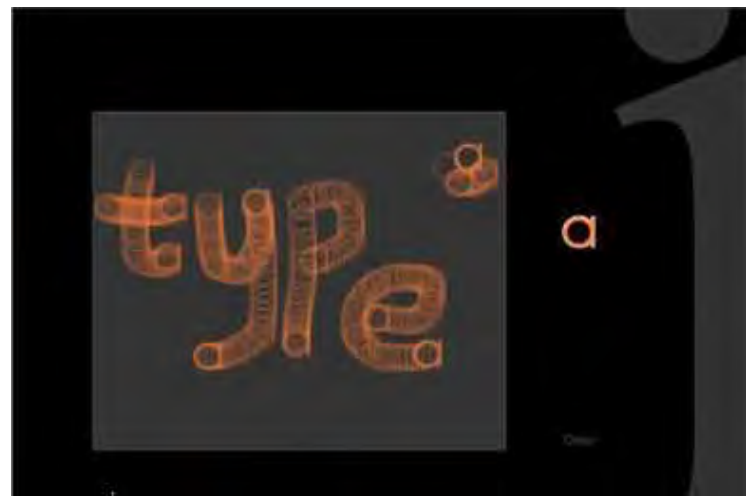

Figure 4. Inspiring 
EVA 2009 London Conference 6-8 July

Anastasios Maragiannis

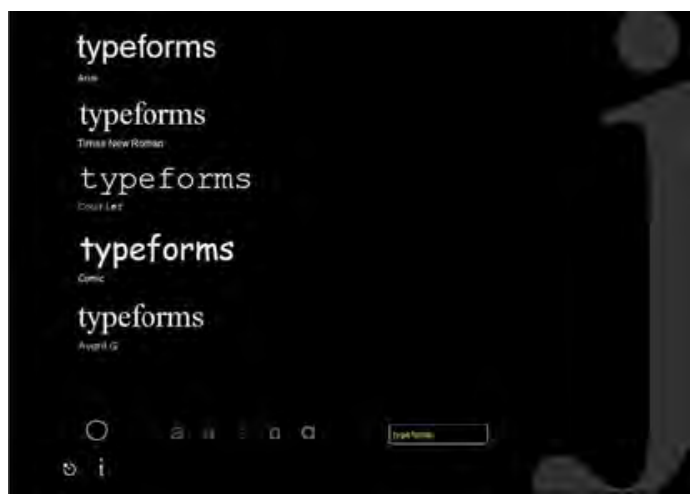

Figure 5. Differentiating

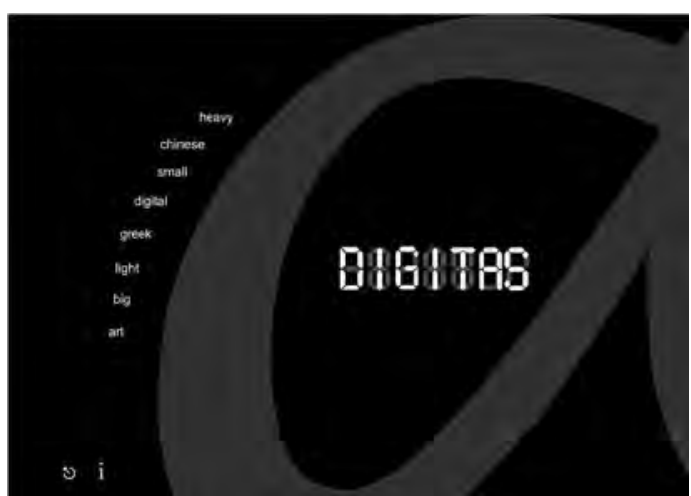

Figure 7. Learning

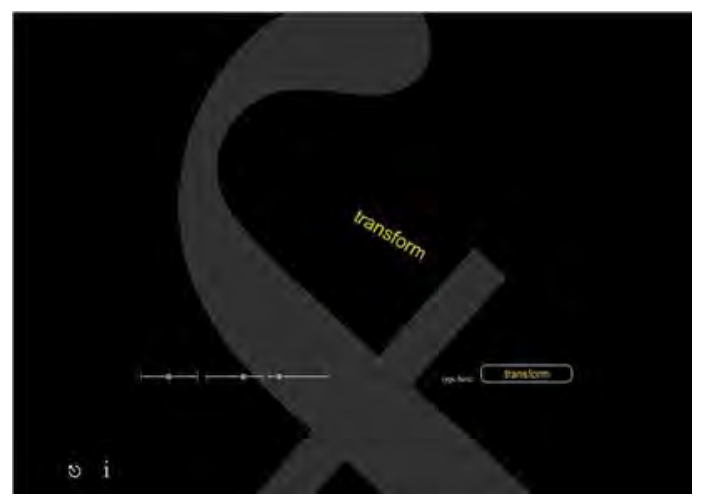

Figure 6. Transforming

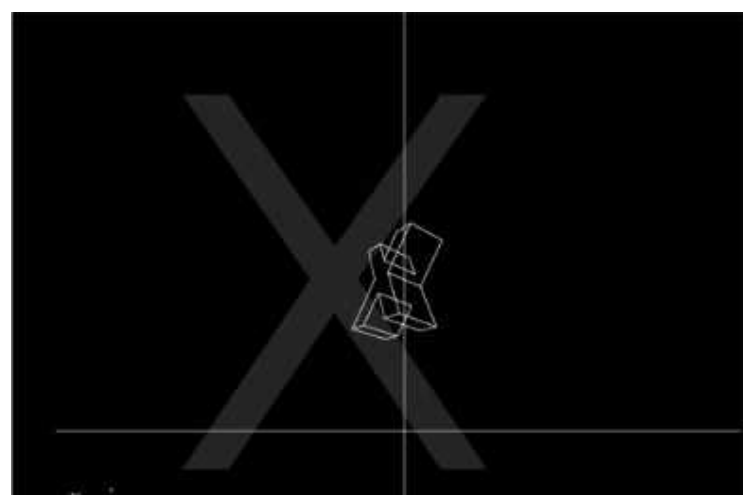

Figure 8. 3D Experience

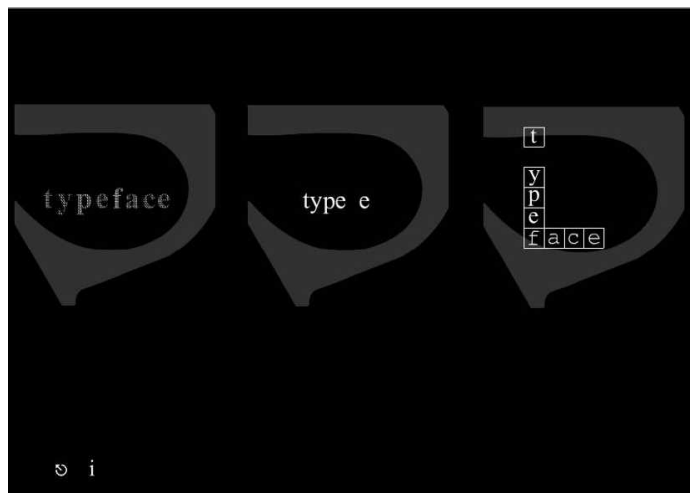

Figure 9. Play

\section{References}

[1] BIGELOW, Charles and DAY, Donald. Digital typography. Scientific American. 249:2 (August 1983). p.106

[2] RUBINSTEIN, Richard. Digital typography : an introduction to type and composition for computer system design. Reading, MA : Addison Wesley, 1988.

[3] KNUTH, Donald E. Computer modem typefaces. Reading, MA : Addison Wesley, 1986. 
EVA 2009 London Conference 6-8 July

Anastasios Maragiannis

[4] RUBINSTEIN, Richard. Digital typography : an introduction to type and composition for computer system design. Reading, MA : Addison Wesley, 1988. pp.141-145.

[5] BIGELOW, Charles and HOLMES, Kris. The design of Lucida(R) : an integrated family of types for electronic literacy. In : J.C. van Vliet Text Processing and Document Manipulation. Proceedings of the International Conference, University of Nottingham, April 14-16, 1986. Cambridge : Cambridge University Press, 1986. pp.1-17.

[6] VANDERLANS, Rudy, LICKO, Zuzanna and GRAY, Mary E. Emigré : graphic design into the digital realm. New York : Van Nostrand Reinhold, 1993. pp.18-25.

[7] GREIMAN, April. Hybrid imagery : the fusion of technology and graphic design. New York: Watson-Guptill Publications, 1990. pp.55-99.

[8] STAPLES, Staples. What happens when the edges dissolve? Eye 5:18 (Autumn 1995) pp.6-7.

[9] ELLISON, A. Digital type. London : Axis 2006. p.136.

[10] HELFAND, Jessica. Electronic typography : the new visual language In : Jessica Helfand Screen : essays on graphic design, new media, and visual culture. New York : Princeton Architectural Press, 2001.

[11] LUPTON, E. Deconstruction and graphic design : history meets theory. Design Writing Research, 1994.

[12] http://maxbruinsma.nl/index1.html

[13] MACDA, John. Flying letters. Tokyo : Naomi Enama, Digitalogue Co., Ltd., 1996.

[14] MACDA, John. 12 o'clocks. Tokyo : Naomi Enama, Digitalogue Co., Ltd., 1997.

[15] MILLER, A. Dimensional typography : case studies on the shape of letters in virtual environments. New York : Princeton Architectural Press, 1996. pp.24-5

[16] Web Designer, Issue 121 p.29.

[17] WOOLMAN, M. and BELLANTONI, J. Moving type : designing for time and space. Rotovision, 2000. p.7.

[18] ibid. 